\title{
Subacute Progressive Ascending Myelopathy from L2 to C4 after A Burst Fracture of the Second Lumbar Vertebra
}

\author{
Sylvia Farzi ${ }^{1 *}$, Gernot Wildner ${ }^{1}$, Rainer Gumpert ${ }^{2}$ and Gerhard Prause ${ }^{1}$
}

${ }^{1}$ Department of Anesthesiology and Critical Care, Medical University of Graz, Graz, Austria

${ }^{2}$ Department of Trauma Surgery, Medical University of Graz, Graz, Austria

\begin{abstract}
Subacute progressive ascending myelopathy is a rare, poorly understood neurological complication of spinal cord injury, unrelated to mechanical compression, instability, or syrinx formation at the level of injury or above. To date, there is no known treatment for this dramatic spinal cord injury complication. We present a case of subacute progressive myelopathy after lumbar spine trauma. The therapy consisted of plasmapheresis, hyperbaric oxygen, high-dose cortisol, antibiotic, and antiviral drugs. At 1 year post injury, the patient had recovered most of his lost upper-extremity function and MRI demonstrated only discrete signal intensity alterations extending to T3/4.
\end{abstract}

Keywords: Spinal cord injury; Fracture fixation; Plasmapheresis; Hyperbaric oxygenation; Necrosis; Apoptosis

\section{Introduction}

Subacute progressive ascending myelopathy (SPAM) is a rare, poorly understood neurological complication of spinal cord injury (SCI) [1-6], unrelated to mechanical compression, instability, or syrinx formation at the level of injury or above. Patients typically present with an ascending neurological deficit within 4 weeks after the initial injury. Characteristic magnetic resonance imaging (MRI) changes are cord expansion and an increased signal on $\mathrm{T}_{2}$-weighted sequences with a medullary distribution [6]. Only one case describing myelopathy ascending as many as 17 levels has been described in the literature [2]. To date, there is no known treatment for this dramatic SCI complication.

\section{Case Presentation}

A 27-year-old man sustained a complex fracture of the second lumbar vertebra with dura mater and nerve root injury after crashing into a tree in a skiing accident. Initial neurological examination showed incomplete conus/cauda syndrome. The cervical and thoracic spine was clinically and radiologically normal. The patient did not have any other injuries. A single dose of methylprednisolone $30 \mathrm{mg} /$ $\mathrm{kg}$ was administered in the field. Decompression, removal of a bone fragment from the spinal canal, dural tear repair, and posterior surgical stabilization from L1 to L3 using USS instrumentation with placement of pedicle screws and an iliac crest bone graft were performed on the day of the injury. Postoperative computed tomography (CT) showed a misplaced screw in the right pedicle at L1 and the patient underwent revision on the next day. The immediate postsurgical period was uneventful. On posttrauma day 3 , neurological examination revealed complete paraplegia at $\mathrm{T} 12$ right and $\mathrm{L} 1$ left. $\mathrm{A} \mathrm{T}_{2}$-weighted MR image showed increased signal intensity within the center of the spinal cord from L2 to T11 as well as a hypointense lesion measuring 10x4 mm at level L1, suspect of an intramedullary hematoma. Six days posttrauma, the paraplegic level had ascended to T10. The patient had not been mobilized before. MRI demonstrated high signal intensity on $\mathrm{T}_{2}$ weighted images extending centrally from L2 to T7. CT angiography ruled out aortic dissection, but was inconclusive with regard to perfusion. Starting on posttrauma day 7 , temperature peaks were observed with the highest peak being 37.8 degrees centigrade. Blood cultures, chest $\mathrm{x}$-ray, and urine test were negative. Leukocyte count was within the normal range and CRP was negative. Consequently, the subfebrile temperature was interpreted as resorption fever. Over the next days, the patient suffered progressive neurological deterioration. On posttrauma day 12, the paraplegic level had ascended to T6/7 and the increased $\mathrm{T}_{2}$-weighted MRI signal in the central cord region extended to T6 (Figure 1). There was also a new area of increased signal intensity at T12 and L1, suggestive of degraded blood products. On posttrauma day 13, the patient was transferred to our institution.

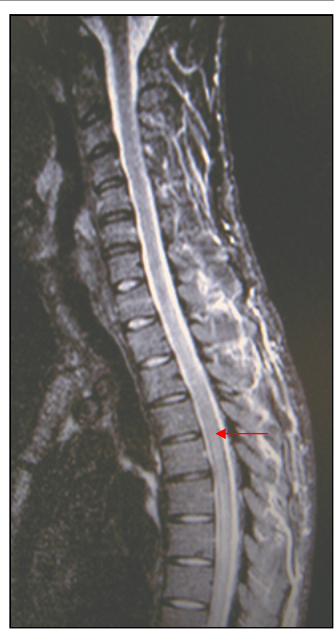

Figure 1: Increased signal intensity reaching T6 (posttrauma day 12)

*Corresponding author: Sylvia Farzi, Department of Anesthesiology and Intensive Care, Hospital of the University of Graz, Auenbruggerplatz 29, 8036 Graz, Austria, Tel: +43-316-385-81102; Fax: +43-316-385-4664; E-mail: sylvia.farzi@medunigraz.at

Received November 10, 2011; Accepted January 10, 2012; Published January 15,2012

Citation: Farzi S, Wildner G, Gumpert R, Prause G (2012) Subacute Progressive Ascending Myelopathy from L2 to C4 after A Burst Fracture of the Second Lumbar Vertebra. J Trauma Treatment 1:109. doi:10.4172/2167-1222.1000109

Copyright: (c) 2012 Farzi S, et al. This is an open-access article distributed under the terms of the Creative Commons Attribution License, which permits unrestricted use, distribution, and reproduction in any medium, provided the original author and source are credited. 
Citation: Farzi S, Wildner G, Gumpert R, Prause G (2012) Subacute Progressive Ascending Myelopathy from L2 to C4 after A Burst Fracture of the Second Lumbar Vertebra. J Trauma Treatment 1:109. doi:10.4172/2167-1222.1000109

The neurological examination revealed a paraplegic level of $\mathrm{T} 5 / 6$, the patient also suffered from burning pain in the dermatomes $\mathrm{T} 1 / 2$ on both sides. On posttrauma day 14, MRI of the spine showed high signal on $\mathrm{T}_{2}$-weighted images extending from the conus to C6/7 (Figure 2). Cerebrospinal fluid (CSF) analysis revealed barrier disruption and a moderate, predominantly monocytic reactive pleocytosis with some scattered macrophages, and was interpreted as being consistent with necrotizing myelopathy. Hyperbaric oxygen (HBO) therapy and highdose methylprednisolone were started. Neurological deterioration continued further with ascension of hypasthesia to the upper extremities. The combination of slowly developing quadriplegia and increased CSF protein led to the suspected diagnosis of Guillain-Barré syndrome and plasmapheresis cycles were initiated. On posttrauma day 17, spinal MRI including angiography was performed. The increased $\mathrm{T}_{2}$-weighted signal in the central cord region now reached C4 (Figure 3). $\mathrm{T}_{1}$ weighted images demonstrated heterogeneous intramedullary signal, the spinal canal was dilated at level T1-2. There was inhomogenous contrast enhancement at level T8-T11/12 after gadolinium application. The MRA showed no evidence of thrombosis, vascular anomaly, or dural arteriovenous fistula. An MRI of the neurocranium was normal. On posttrauma day 18, the patient had a complete sensory level at C5. Extensive virological, serological, and immunological screening was negative. In addition to 5 cycles of plasmapheresis, HBO therapy, and high-dose cortisol, antiviral (acyclovir) and antibiotic drugs were administered. 4 weeks post trauma, the extension of the characteristic signal alterations persisted at level C4 on MRI and the patient showed only minimal improvement of neurological symptoms. At 1 year post injury, he had recovered most of his lost upper-extremity function and MRI demonstrated only discrete signal intensity alterations extending to T3/4. The latest follow-up, at 3 years post injury, showed further clinical improvement of neurological function with sensory level at T4 and motor level at T6. The 3-year-follow-up MRI showed regression of signal alterations to T4, pronounced myelon atryophy below T4, and bone fusion at L1/2 level (Figure 4).

\section{Discussion}

The syndrome of delayed, subacute neurological deterioration by four or more segments following traumatic spinal cord injury was

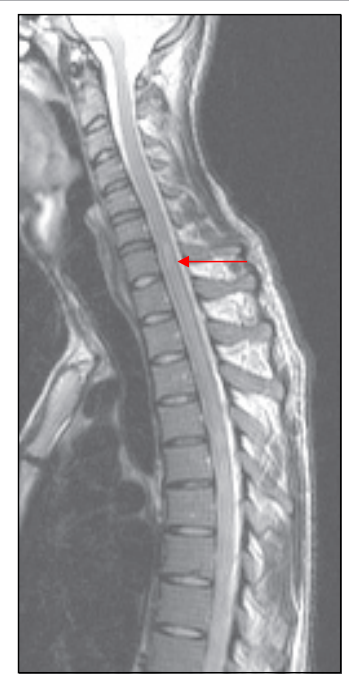

Figure 2: Increased signal intensity reaching C6/7 (posttrauma day 14).

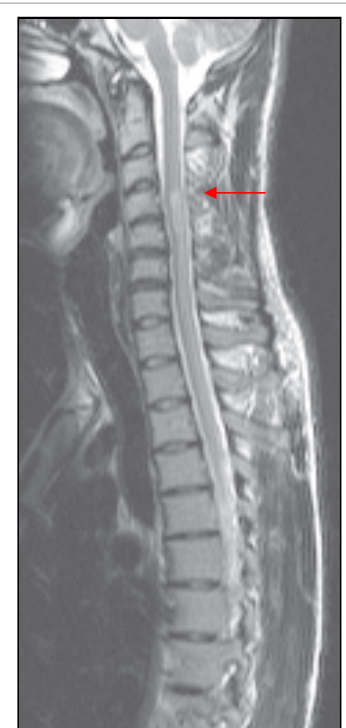

Figure 3: Increased signal intensity reaching C4 (posttrauma day 17).

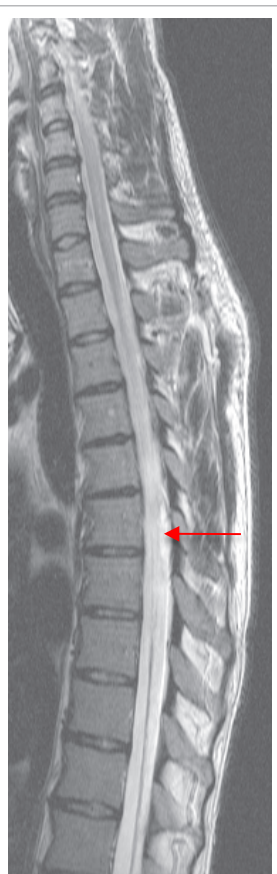

Figure 4: Follow-up at 3 years: atrophic myelon below T4.

first described by Frankel in 1968, who identified eight patients, an estimated incidence of $1.5 \%$ of all spinal cord injuries [1]. A total of only 39 cases [1-7] of SPAM have been published to date, indicating that it is either exceedingly rare or grossly underreported. In particular, the occurrence of subacute delayed myelopathy after low spinal injury seems to be very rare. The only other case describing myelopathy ascending as many as 17 levels after low spinal injury (L1 level) was reported by Belanger [2]. Several hypotheses have been put forth to explain the origin of this syndrome [2].

The arterial hypothesis assumes that anterior spinal cord artery [8] thrombosis is a potential cause of the delayed deterioration. If this had been the case in our patient, deterioration would have occurred 
abruptly without daily progression and the posterior column would have been preferentially spared. However, the pattern on axial MR imaging was central and thus did not appear to respect a classic ASA distribution.

Venous thrombosis and congestive ischemia are another possible etiological mechanism in ascending myelopathy. None of the MR images in our patient demonstrated venous stasis or engorgement of surface venous structures.

Ischemia due to hypotension has been proposed as a potential mechanism for SPAM [7]. This will, however, not explain the progressive nature of the ascending myelopathy unless there are repeated or sustained hypotensive episodes. Specifically, our patient was hemodynamically stable with no reported episodes of hypotension.

Inflammatory or autoimmune reaction of the central nervous system (CNS) is another possible etiological mechanism of SPAM. It could be argued that such a response may be triggered by a traumatic event, although a distinct association between the two conditions has never been identified. Our patient, however, was extensively tested for inflammatory and autoimmune processes and all the results were negative. No involvement of other parts of the CNS was noted, which could be expected in cases of demyelinating disease.

In our patient, there was no elevated leukocyte count or CRP value, no pyrexia, CSF analysis showed no signs of infection, and virological and serological workup was negative, virtually ruling out infective myelitis as a possible etiological factor.

SCI injury triggers a complex cascade of secondary neurodegenerative phenomena that are set on by the primary injury. These secondary events include neurogenic shock, vascular insults such as hemorrhage and ischemia-reperfusion, lipid peroxidation, inflammation, excitotoxicity, intracellular calcium increment, apoptosis, and disturbance of mitochondrial function [9]. The possible role of apoptosis in the causation and progression of SPAM has been elucidated by Al-Ghatany et al. [3]. While there is now strong morphological and biochemical evidence of the presence of apoptosis after SCI [8,10-20], even at distances remote from the area of initial injury [12,15,21] and persisting for many weeks [12,14,15], the mediators of post-SCI apoptosis are not yet well understood.

A number of pharmacological neuroprotective agents targeting one or more of the known secondary injury events have been studied. Some of them have shown benefit in experimental and even in clinical trials [9]. However, they are with the exception of methylprednisolone (MP) not ready for adoption in the management of patients. MP is commonly used in the setting of acute SCI based on the results of randomized controlled trials [22,23]. However, many authors have questioned the efficacy of MP because of its marginal benefits and criticisms of study design and interpretation [24-27]. MP's mechanism of action has been attributed to anti-inflammatory or antioxidant properties. Interestingly, recently published findings suggest that MP may have direct neuroprotective activities, selectively inhibiting oligodendrocyte but not neuronal cell death via receptor-mediated action [28].

\section{Conclusion}

In summary, it is conceivable that the delayed neurological deterioration seen in our patient was due to uncontrolled secondary injury mechanisms including necrosis and apoptosis. Whether the ex iuvantibus treatment with high-dose MP and HBO contributed to the regression of neurologic symptoms in our patient can only be speculated. In any case, it has been reported that hyperbaric oxygen accelerates neurologic recovery after spinal cord injury by ameliorating mitochondrial dysfunction in the motor cortex and spinal cord, arresting the spread of hemorrhage, reversing hypoxia, and reducing edema [29].

\section{References}

1. Frankel HL (1969) Ascending cord lesion in the early stages following spinal injury. Paraplegia 7: 111-118.

2. Belanger E, Picard C, Lacerte D, Lavallee P, Levi AD (2000) Subacute posttraumatic ascending myelopathy after spinal cord injury. Report of three cases. J Neurosurg 93: 294-299.

3. Al-Ghatany M, Al-Shraim M, Levi AD, Midha R (2005) Pathological features including apoptosis in subacute posttraumatic ascending myelopathy. Case report and review of the literature. J Neurosurg Spine 2: 619-623.

4. Schmidt BJ (2006) Subacute delayed ascending myelopathy after low spine injury: case report and evidence of a vascular mechanism. Spinal Cord 44 : 322-325.

5. Yablon IG, Ordia J, Mortara R, Reed J, Spatz E (1989) Acute ascending myelopathy of the spine. Spine 14: 1084-1089.

6. Planner AC, Pretorius PM, Graham A, Meagher TM (2008) Subacute progressive ascending myelopathy following spinal cord injury: MRI appearances and clinical presentation. Spinal Cord 46: 140-144.

7. Aito S, El Masry WS, Gerner HJ, Lorenzo ND, Pellicano G, et al. (1999) Ascending myelopathy in the early stage of spinal cord injury. Spinal Cord 37 : 617-623.

8. Emery E, Aldana P, Bunge MB, Puckett W, Srinivasan A, et al. (1998) Apoptosis after traumatic human spinal cord injury. J Neurosurg 89: 911-920.

9. Martinon S, Ibarra A (2008) Pharmacological neuroprotective therapy for acute spinal cord injury: state of the art. Mini Rev Med Chem 8: 222-230.

10. Beattie MS, Farooqui AA, Bresnahan JC (2000) Review of current evidence for apoptosis after spinal cord injury. J Neurotrauma 17: 915-925.

11. Beattie MS, Hermann GE, Rogers RC, Bresnahan JC (2002) Cell death in models of spinal cord injury. Prog Brain Res 137: 37-47.

12. Crowe MJ, Bresnahan JC, Shuman SL, Masters JN, Beattie MS (1997) Apoptosis and delayed degeneration after spinal cord injury in rats and monkeys. Nat Med 3: 73-76.

13. Hains BC, Black JA, Waxman SG (2003) Primary cortical motor neurons undergo apoptosis after axotomizing spinal cord injury. J Comp Neurol 462 $328-341$.

14. Keane RW, Kraydieh S, Lotocki G, Bethea JR, Krajewski S, et al. (2001) Apoptotic and anti-apoptotic mechanisms following spinal cord injury. J Neuropathol Exp Neurol 60: 422-429.

15. Li GL, Farooque M, Holtz A, Olsson Y (1999) Apoptosis of oligodendrocytes occurs for long distances away from the primary injury after compression trauma to rat spinal cord. Acta Neuropathol 98: 473-480.

16. Liu XZ, Xu XM, Hu R, Du C, Zhang SX, et al. (1997) Neuronal and glia apoptosis after traumatic spinal cord injury. J Neurosci 17: 5395-5406.

17. Lu J, Ashwell KW, Waite P (2000) Advances in secondary spinal cord injury: role of apoptosis. Spine 25: 1859-1866.

18. Takagi T, Takayasu M, Mizuno M, Yoshimoto M, Yoshida J (2003) Caspase activation in neuronal and glial apoptosis following spinal cord injury in mice. Neurol Med Chir (Tokyo) 43: 20-29.

19. Wingrave JM, Schaecher KE, Sribnick EA, Wilford GG, Ray SK, et al. (2003) Early induction of secondary injury factors causing activation of calpain and 
Citation: Farzi S, Wildner G, Gumpert R, Prause G (2012) Subacute Progressive Ascending Myelopathy from L2 to C4 after A Burst Fracture of the Second Lumbar Vertebra. J Trauma Treatment 1:109. doi:10.4172/2167-1222.1000109

mitochondria-mediated neuronal apoptosis following spinal cord injury in rats. J Neurosci Res 73: 95-104.

20. Zurita M, Vaquero J, Oya S, Morales C (2002) Effects of dexamethasone on apoptosis-related cell death after spinal cord injury. J Neurosurg 96: 83-89.

21. Warden P, Bamber NI, Li H, Esposito A, Ahmad KA, et al. (2001) Delayed glial cell death following wallerian degeneration in white matter tracts after spinal cord dorsal column cordotomy in adult rats. Exp Neurol 168: 213-224.

22. Bracken MB, Shepard MJ, Holford TR, Leo-Summers L, Aldrich EF, et al. (1997) Administration of methylprednisolone for 24 or 48 hours or tirilazad mesylate for 48 hours in the treatment of acute spinal cord injury. Results of the Third National Acute Spinal Cord Injury Randomized Controlled Trial. National Acute Spinal Cord Injury Study. JAMA 277: 1597-1604.

23. Bracken MB, Shepard MJ, Collins WF, Holford TR, Young W, et al. (1990) A randomized, controlled trial of methylprednisolone or naloxone in the treatment of acute spinal-cord injury. Results of the Second National Acute Spinal Cord Injury Study. N Engl J Med 322: 1405-1411.

24. Sayer FT, Kronvall E, Nilsson OG (2006) Methylprednisolone treatment in acute spinal cord injury: the myth challenged through a structured analysis of published literature. Spine J 6: 335-343.

25. Coleman WP, Benzel D, Cahill DW, Ducker T, Geisler F, et al. (2000) A critical appraisal of the reporting of the National Acute Spinal Cord Injury Studies (II and III) of methylprednisolone in acute spinal cord injury. J Spinal Disord 13: 185-199.

26. Hurlbert RJ (2000) Methylprednisolone for acute spinal cord injury: an inappropriate standard of care. J Neurosurg 93: 1-7.

27. Nesathurai S (1998) Steroids and spinal cord injury: revisiting the NASCIS 2 and NASCIS 3 trials. J Trauma 45: 1088-1093.

28. Lee JM, Yan P, Xiao Q, Chen S, Lee KY, et al. (2008) Methylprednisolone protects oligodendrocytes but not neurons after spinal cord injury. J Neurosci 28: 3141-3149.

29. Al-Waili NS, Butler GJ, Beale J, Abdullah MS, Hamilton RW, et al. (2005) Hyperbaric oxygen in the treatment of patients with cerebral stroke, brain trauma, and neurologic disease. Adv Ther 22: 659-678. 